\title{
Several specific high-resolution computed tomography patterns correlate with survival in patients with idiopathic pulmonary fibrosis
}

\author{
Minna E. Mononen ${ }^{1,2}$, Hannu-Pekka Kettunen ${ }^{3}$, Sanna-Katja Suoranta ${ }^{3}$, Miia S. Kärkkäinen ${ }^{4}$, \\ Tuomas A. Selander ${ }^{5}$, Minna K. Purokivi ${ }^{2 \#}$, Riitta L. Kaarteenaho ${ }^{\text {\#\# }}$ \\ ${ }^{1}$ Division of Respiratory Medicine, Institute of Clinical Medicine, School of Medicine, Faculty of Health Sciences, University of Eastern Finland, \\ Kuopio, Finland; ${ }^{2}$ Center of Medicine and Clinical Research, Division of Respiratory Medicine, Kuopio University Hospital, Kuopio, Finland; \\ ${ }^{3}$ Department of Clinical Radiology, Kuopio University Hospital, Kuopio, Finland; ${ }^{4}$ Kuopio City Home Care, Rehabilitation and Medical Services for \\ Elderly, Kuopio, Finland; ${ }^{5}$ Science Services Center, Kuopio University Hospital, Kuopio, Finland; ${ }^{6}$ Research Unit of Internal Medicine, University \\ of Oulu and Medical Research Center Oulu, Oulu University Hospital, Oulu, Finland \\ Contributions: (I) Conception and design: RL Kaarteenaho, MK Purokivi; (II) Administrative support: RL Kaarteenaho, MK Purokivi; (III) Provision \\ of study materials or patients: RL Kaarteenaho, MK Purokivi, MS Kärkkäinen, HP Kettunen, SK Suoranta; (IV) Collection and assembly of data: \\ MS Kärkkäinen, HP Kettunen, SK Suoranta, ME Mononen; (V) Data analysis and interpretation: RL Kaarteenaho, MK Purokivi, ME Mononen, \\ TA Selander; (VI) Manuscript writing: All authors; (VII) Final approval of manuscript: All authors. \\ \#These authors contributed equally to this work. \\ Correspondence to: Minna E. Mononen, MD. Center of Medicine and Clinical Research, Division of Respiratory Medicine, Kuopio University \\ Hospital, POB 100, 70029 Kuopio, Finland. Email: miemonon@uef.fi.
}

Background: Evidence of honeycombing in high-resolution computed tomography (HRCT) is a recognized risk factor for shortened survival in patients with idiopathic pulmonary fibrosis (IPF), but few studies have evaluated the feasibility of exploiting other specific patterns for predicting survival. The aim of this study was to examine the extent of specific HRCT patterns in IPF and determine whether they correlate with clinical features, pulmonary function tests (PFT), and survival.

Methods: Both the presence and extent of specific HRCT patterns, such as traction bronchiectasis, honeycombing, architectural distortion, reticulation, emphysema, and ground glass opacity, in 129 HRCT examinations were scored semi-quantitatively in three zones of each lung. HRCT examinations were also reclassified according to the 2011 and 2018 international statements. Correlations were calculated between the scores of specific HRCT patterns, clinical features, PFT, and patient survival.

Results: The extent of traction bronchiectasis was found to be an independent risk factor of shortened survival (HR 1.227, $\mathrm{P}=0.001$ ). Patients with a possible usual interstitial pneumonia (UIP) pattern had a better median survival than the patients with a definite UIP pattern (61 vs. 37 months, $\mathrm{P}=0.026$ ). The extents of traction bronchiectasis, honeycombing, and architectural distortion displayed an inverse correlation with all PFT values at the time of diagnosis. There were few differences between the radiological classifications of the 2011 and 2018 international statements.

Conclusions: We conclude that several specific HRCT patterns displayed a correlation with shortened survival in IPF; these may help in evaluating the risk of death in IPF patients.

Keywords: Idiopathic pulmonary fibrosis (IPF); radiology; survival

Submitted May 17, 2020. Accepted for publication Mar 03, 2021.

doi: $10.21037 /$ jtd-20-1957

View this article at: http://dx.doi.org/10.21037/jtd-20-1957

(c) Journal of Thoracic Disease. All rights reserved. 


\section{Introduction}

Idiopathic pulmonary fibrosis (IPF) is a heterogeneous disease that has a variable course, which makes predicting survival challenging (1). High-resolution computed tomography (HRCT) is the most important noninvasive diagnostic method in the diagnosis of IPF, but the feasibility of exploiting HRCT for evaluating the course of the disease remains unclear. In addition, several previous studies investigating specific HRCT patterns in IPF, diagnosed according to the 2011 international statement, have mainly concentrated on diagnosis (2-4).

Honeycombing is considered as one of the core findings when the UIP pattern is diagnosed in HRCT (5). Many of the studies comparing disease progression with HRCT findings have concentrated on the honeycombing pattern, revealing its association with shortened survival in IPF (6-8). Patients with a honeycombing score higher than $15 \%$ in HRCT have been shown to have a shorter survival than those with a honeycombing score less than $15 \%$ (6). In a recent study of 350 IPF patients, honeycombing was associated with poorer survival (7) and it has also been reported that honeycombing was associated with an increased risk of death (8).

The previous studies investigating either the presence or extent of traction bronchiectasis for predicting survival in IPF patients who have been diagnosed according to 2011 international IPF statement, have shown discrepant results (7-9). In the study of Arcadu et al., the presence of traction bronchiectasis was not associated with shortened survival, unlike the global assessment of interstitial abnormalities (7). Salisbury et al. found that the fibrosis score (number of lobes where traction bronchiectasis was present) was associated with an increased risk of death (8). Recently, Sverzellati et al. found that the extent of traction bronchiectasis was associated with shortened survival (9).

Since relatively little is known about the correlation between specific HRCT patterns (except honeycombing) and survival in IPF patients diagnosed according to the 2011 international statement, we aimed to evaluate both the presence and extent of specific HRCT patterns and to examine their correlation with clinical features, such as age, gender, smoking history, dyspnea, cough, long term oxygen therapy, and the number of hospitalizations due to respiratory reasons, as well as data from pulmonary function tests (PFT), and finally with patient survival. Furthermore, we have classified the HRCT examinations according to both the 2011 and 2018 international statements in order to determine whether the application of different guidelines would change the radiological categorization $(1,5)$. We present the following article in accordance with the STROBE reporting checklist (available at http://dx.doi. org/10.21037/jtd-20-1957).

\section{Methods}

\section{Patient selection and data collection}

The subjects of this study were obtained from Kuopio University Hospital pulmonology clinic medical records (digitized from 2004 onwards) from January 2002 to December 2012 using the International Classification Codes (ICD-10) J84.1, J84.8 and J84.9. From the 610 patients discovered, 132 IPF-patients were identified from their clinical information, radiology, and histology when available, and accepted after reaching a multidisciplinary consensus. Patients with pulmonary fibrosis caused by a known reason, for example, asbestosis or connective tissue disease, were excluded. Smoking history, clinical information, and PFT were retrospectively gathered. Death or lung transplantation was considered as the outcome. The collection of this data has been described in more detail in three previous publications (10-12).

Because of the retrospective nature of this study and the fact that most of the study subjects were already deceased, no informed consents for study inclusion were collected. The study protocol was approved by the Research Ethics Committee of the Northern Savo Hospital District (statement 17/2013) and by the National Institute for Health and Welfare (Dnro THL/1052/5.05.01/2013). This study was conducted in compliance with the Declaration of Helsinki (as revised in 2013).

\section{The CT protocol}

Digital HRCT scans and analogue film images, at the time of diagnosis, were available from 99 of 132 patients $(75 \%)$ and 28 patients $(21 \%)$, respectively. Other comparable CT examinations, such as CT pulmonary angiography, were available from five patients. All HRCT scans were spaced axial scans. The radiologists evaluated the material other than digital HRCT scans eligible for further analysis. A total of 86 out of 132 images (65\%) were evaluated as good quality, 43 as suboptimal quality, and three as poor quality/ not diagnostic. The poor-quality images were excluded, and thus the final number of study subjects was 129, of which 97 
(75\%) had undergone digital HRCT scanning.

\section{Re-classifying of CT}

Two radiologists independently re-classified the HRCT scans according to the 2011 international statement into three classes: usual interstitial pneumonia (UIP), possible UIP, and inconsistent with UIP (1). The first radiologist (10 years of expertise in thoracic radiology) re-classified the 129 HRCT examinations in 2014. In 2018, the second radiologist (2 years of expertise in thoracic radiology) re-classified 121 of the original HRCT examinations available according to both the 2011 and 2018 international statements i.e., as UIP, probable UIP, indeterminate with UIP, and alternative diagnosis (5). There were no differences in the categorical classification of the HRCT scans between good and suboptimal quality HRCTs.

\section{Scoring of specific HRCT patterns}

The first radiologist assessed the 129 HRCT examinations in detail using a form designed for a previous study (13). The presence and extent of several specific HRCT patterns such as honeycombing, traction bronchiectasis, emphysema, architectural distortion, reticulation, ground-glass opacity (GGO), pleural plaques, consolidation, bronchiectasis, and crazy paving were assessed separately in three zones of each lung. The extents of GGO, reticulation, and honeycombing were semi-quantitatively graded on a scale of 0 to $4(0=$ absent, $1=$ minor peripheral scattered changes, $2=$ uniform peripheral or minor central scattered changes, $3=$ substantial peripheral changes that penetrated deeply into the lung parenchyma, $4=$ very abundant peripheral and central changes). Instead, emphysema, traction bronchiectasis, and architectural distortion were scored using a scale of 0 to $3(0=$ absent, $1=$ single scattered changes, $2=$ larger single or several minor changes, $3=$ uniform or substantial changes) (Figure 1). Scores from all six zones were summed to obtain the total score for each of the patterns, the maximum scores being 24 or 18 , respectively. The scoring system has been described in more detail in a previous publication (13). To assess the repeatability of the scoring, the second radiologist scored 26 of the original HCRT examinations, of which $96 \%$ were good and only $4 \%$ were of suboptimal quality. There were mainly no differences in specific HRCT patterns observed between good and suboptimal quality HRCT examinations, except for traction bronchiectasis. There were more HRCT examinations where traction bronchiectasis was observed among the good quality HRCTs than among the suboptimal quality HRCTs (91\% vs. 67\%, respectively, $\mathrm{P}=0.001$ ).

\section{Pulmonary function tests}

The PFT values at the time of diagnosis are presented as percent predicted. The change in forced vital capacity (FVC) and diffusion capacity to carbon monoxide (DLCO) during six and 12 months' follow-up have been calculated as follows: (FVC or DLCO at 6 or 12 months-FVC or $\mathrm{DLCO}$ at the time of diagnosis)/FVC or DLCO at the time of diagnosis $\times 100$, with the result expressed in percentages. Furthermore, the FVC change during 6 and 12 months was classified into two categories: $<10 \%$ decline or improvement of FVC and $\geq 10 \%$ decline in FVC. Similarly, DLCO was classified into two categories using a $15 \%$ decline as a cutoff point (14).

\section{Statistical analysis}

Data were expressed as means and standard deviations with ranges or frequencies with percentages. The presence and extent of specific HRCT patterns, clinical features, PFT and survival were analyzed in the different HRCT categories according to the 2011 international statement (1). Independent-samples Kruskal-Wallis or Mann-Whitney $\mathrm{U}$ tests were used for continuous variables, with Chisquare test also being used when appropriate. Correlations between variables were investigated with the Spearman rank correlation coefficient. The Kaplan-Meier estimator and Cox regression analysis were used to evaluate the association between variables and survival. Clinical features such as age, gender, PFT, FVC and DLCO absolute change for 12 months, oxygen therapy, dyspnea, cough, and smoking status, as well as specific HRCT patterns were analyzed in a univariate Cox regression analysis. Some of the data of the clinical features has been published previously (12). Thereafter, a stepwise Cox regression model was built using variables with univariate $\mathrm{P}$ values $<0.20$, entering variables at the 0.05 significance level and removing them at the 0.10 level. Inter-observer agreements of the HRCT examinations according to the 2011 international statement and the specific HRCT patterns are presented as a kappa $(\kappa)$ value: good agreement $\kappa=0.61-0.80$, moderate agreement $\kappa=0.41-0.60$ and fair agreement $\kappa=0.21-0.40$. $P$ values $<0.05$ were considered statistically significant. Missing data was excluded. IBM statistics SPSS software, version 22.0, was 

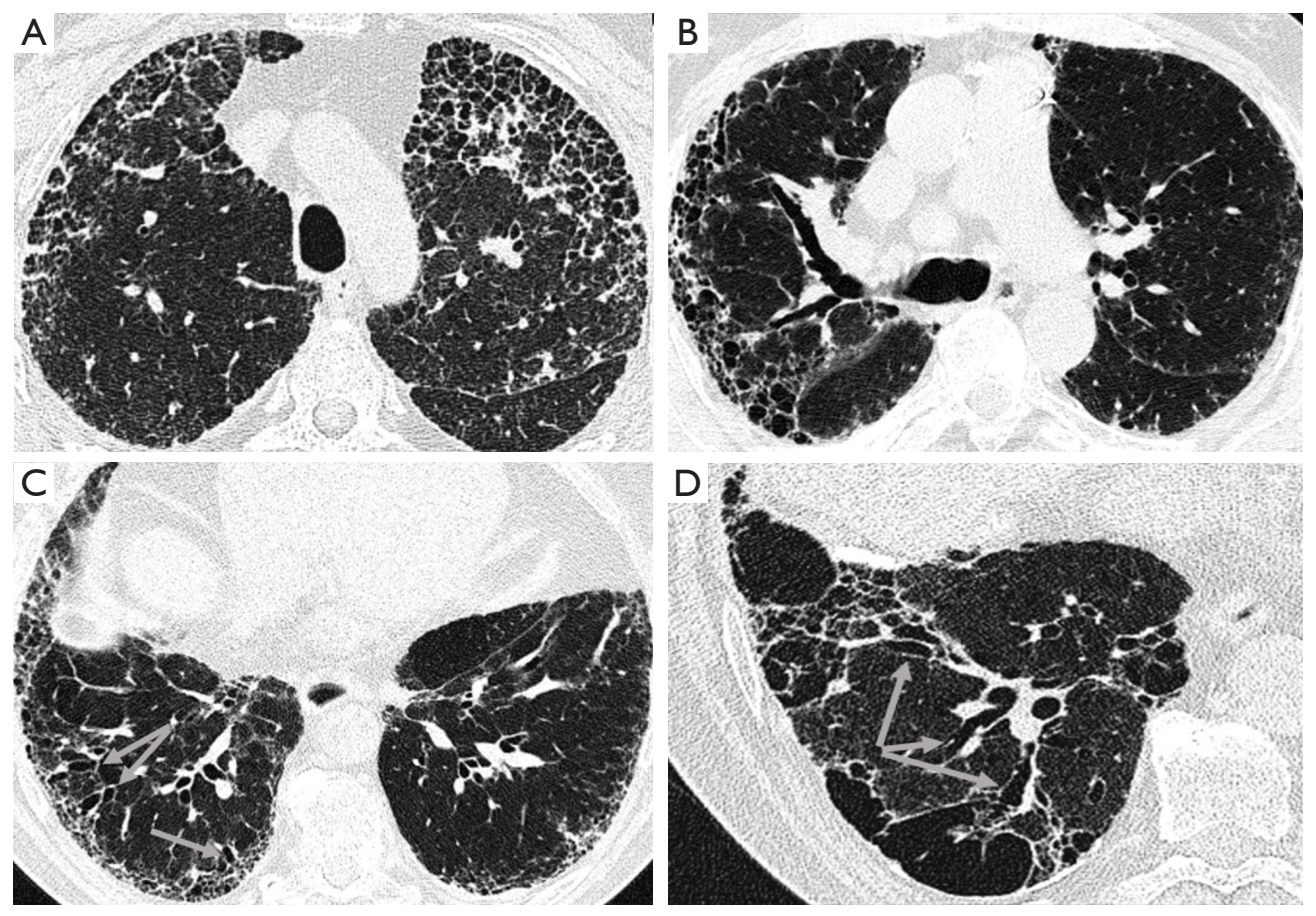

Figure 1 Representative HRCT images from four UIP patients with emphysema and fibrosis, categorized according to the 2018 international statement. (A) HRCT shows uniform peripheral scattered changes (score 2) of honeycombing in the right upper zone and substantial signs of peripheral honeycombing that penetrated deeply into the lung parenchyma (score 3) in the left upper zone; (B) HRCT shows minor peripheral scattered changes (score 1) of reticulation in the middle zones and uniform peripheral scattered changes (score 2) of honeycombing on the right side, as well as minor peripheral scattered changes (score 1) of honeycombing on the left side; (C) HRCT shows uniform peripheral scattered changes (score 2) of reticulation in the lower zones and larger single scattered changes (score 2) of traction bronchiectasis (arrows), as well as minor peripheral scattered changes (score 1) of honeycombing only on the right side; (D) HRCT reveals larger single scattered changes (score 2) of paraseptal and bullous emphysema in the right lower zone, as well as larger single scattered changes (score 2) of traction bronchiectasis (arrows) and honeycombing. HRCT, high-resolution computed tomography; UIP, usual interstitial pneumonia.

used in the statistical analysis of the whole data.

\section{Results}

\section{HRCT classification according to the 2011 and 2018 international IPF statements}

Sixty-five HRCT examinations were classified as UIP (50\%), 42 as possible UIP (32\%), and 22 as inconsistent with UIP (17\%) according to the 2011 guidelines. There were only subtle differences between the radiological classifications of the 2011 and 2018 international statements, since the UIP category did not change, whereas the HRCT examinations in the possible UIP category were divided mostly into the probable and the indeterminate with UIP categories (Figure 2).

\section{Inter-observer agreement of the radiologists}

The inter-observer agreement of the radiologists was moderate $(\kappa=0.429)$ in the re-classification of the patterns into three categories, i.e., UIP, possible UIP, and inconsistent with UIP. When the UIP pattern was compared with the non-UIP pattern (the possible and inconsistent with UIP patterns combined) inter-observer agreement was higher $(\kappa=0.551)$. Inter-observer agreement was good when evaluating honeycombing $(\kappa=0.783)$ and emphysema $(\kappa=0.623)$.

\section{Patient characteristics and specific HRCT patterns}

The majority of the patients were men $(73 \%)$ with a mean age of 71 years. The characteristics of the patients and the 
A

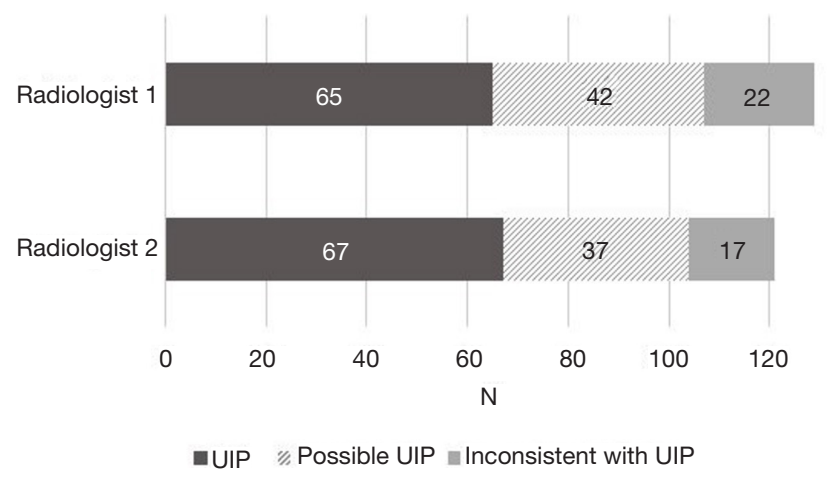

B

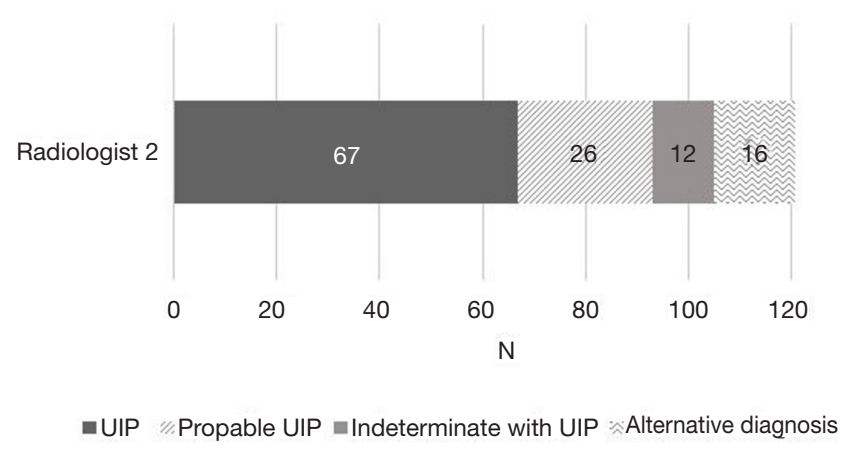

Figure 2 Radiological categorization of HRCT examinations in patients with idiopathic pulmonary fibrosis (IPF) (A) according to the 2011 IPF statement ( $\mathrm{N}=129$ and $\mathrm{N}=121$, respectively) and (B) according to the $2018 \mathrm{IPF}$ statement $(\mathrm{N}=121)$. Numbers are presented as $\mathrm{N}$. HRCT, high-resolution computed tomography; UIP, usual interstitial pneumonia.

Table 1 Characteristics of the patients with idiopathic pulmonary fibrosis categorized according to the $2011 \mathrm{IPF}$ statement

\begin{tabular}{|c|c|c|c|c|}
\hline Feature & All $(\mathrm{N}=129)$ & UIP $(\mathrm{N}=65)$ & Possible UIP (N=42) & Inconsistent with UIP ( $\mathrm{N}=22)$ \\
\hline FVC\%, mean (SD) & $76.7(18.6)$ & 75.7 (19.5) & $79.7(18.4)$ & $74.2(16.6)$ \\
\hline FEV1\%, mean (SD) & $77.1(17.1)$ & $75.9(18.1)$ & $78.9(16.3)$ & $76.8(16.1)$ \\
\hline DLCO\%, mean (SD) & $55.9(17.2)$ & $53.0(14.9)^{\dagger}$ & $63.0(19.3)^{\S}$ & $50.7(15.9)$ \\
\hline Female & 35 [27] & $12[18]^{\ddagger}$ & $11[26]^{\S}$ & $12[54]$ \\
\hline Ever-smoker & $82 / 126[65]$ & $46 / 63[73]^{\ddagger}$ & $25 / 41[61]$ & $11[50]$ \\
\hline Dyspnea & $96[74]$ & $48[74]$ & 29 [69] & 19 [86] \\
\hline Cough & 77 [60] & $34[52]^{\dagger}$ & $30[71]$ & $13[59]$ \\
\hline
\end{tabular}

Numbers are presented as mean (SD) or $\mathrm{N}[\%]$. P value $<0.05{ }^{\dagger}$ UIP vs. possible UIP, ${ }^{\ddagger}$ UIP vs. inconsistent with UIP, ${ }^{\S}$ possible UIP vs. inconsistent with UIP. UIP, usual interstitial pneumonia; FVC\%, forced vital capacity percent predicted; FEV1\%, forced expiratory volume in one second percent predicted; DLCO\%, diffusion capacity to carbon monoxide percent predicted; ReHosP, hospitalization due to respiratory reasons; $\mathrm{N}$, number; $\mathrm{SD}$, standard deviation.

different radiological categories are presented in Table 1 . Some of the data have been published previously (10-12). Reticulation was the most predominant HRCT pattern found in $99 \%$ of cases, followed by traction bronchiectasis (83\%). The extent of traction bronchiectasis was higher in the UIP group than in the other two groups. Tables 2 and 3 present the specific HRCT patterns in the whole cohort and in the different categories.

\section{Correlation of the HRCT patterns with survival}

The mean follow-up time was 50 months (range, 0-188 months). One hundred and twenty-three patients (95\%) died during the follow-up and two patients underwent lung transplantation. The extents of traction bronchiectasis, honeycombing, architectural distortion, and emphysema were associated with shortened survival in a univariate Cox regression analysis (Table 4). A univariate 
Table 2 Specific HRCT patterns of patients with idiopathic pulmonary fibrosis categorized according to the 2011 IPF statement

\begin{tabular}{lcccc}
\hline Radiological pattern & All $(\mathrm{N}=129)$ & UIP $(\mathrm{N}=65)$ & Possible UIP (N=42) & Inconsistent with UIP (N=22) \\
\hline Reticulation & $128[99]$ & $64[99]$ & $42[100]$ & $22[100]$ \\
Traction bronchiectasis & $106 / 128[83]$ & $59[91]^{\dagger}$ & $30 / 41[73]$ & $17[77]$ \\
Honeycombing & $91 / 122[75]$ & $64[99]^{\dagger \ddagger}$ & $18 / 37[47]$ & $12[29]$ \\
Architectural distortion & $63[49]$ & $44[68]^{\dagger \ddagger}$ & $29 / 38[76]$ & $7[32]$ \\
GGO & $61 / 117[52]$ & $16 / 59[27]^{\dagger \ddagger}$ & $6[14]$ & $16 / 20[80]$ \\
Emphysema & $34[26]$ & $24[37]^{\dagger}$ & - & $4[18]$ \\
Plaques & $36 / 128[28]$ & - & - & - \\
Consolidation & $3[2]$ & - & - & - \\
Bronchiectasis & $4[3]$ & - & - \\
Crazy paving & $1[1]$ & - & - \\
\hline
\end{tabular}

Numbers are presented as $\mathrm{N}[\%]$. P value $<0.05{ }^{\dagger}$ UIP vs. possible UIP, ${ }^{\ddagger}$ UIP vs. inconsistent with UIP. HRCT, high-resolution computed tomography; UIP, usual interstitial pneumonia; GGO, ground glass opacity; N, number.

Table 3 The extent of specific HRCT patterns in patients with idiopathic pulmonary fibrosis categorized according to the 2011 IPF statement

\begin{tabular}{lcccc}
\hline Radiological pattern $^{\S}$ & All $(\mathrm{N}=129)$ & UIP $(\mathrm{N}=65)$ & Possible UIP $(\mathrm{N}=42)$ & Inconsistent with UIP $(\mathrm{N}=22)$ \\
\hline Reticulation & $8.9 \pm 3.1(0-19)$ & $8.39 \pm 3.30(0-19)^{\ddagger}$ & $9.14 \pm 2.95(3-16)$ & $10.19 \pm 2.44(5-14)$ \\
Traction bronchiectasis & $3.5 \pm 2.9(0-13)$ & $4.49 \pm 2.94(0-13)^{\dagger \ddagger}$ & $2.22 \pm 2.29(0-11)$ & $2.82 \pm 2.58(0-10)$ \\
Honeycombing & $3.6 \pm 3.5(0-14)$ & $5.77 \pm 3.22(1-14)^{\dagger \ddagger}$ & $1.11 \pm 1.61(0-8)$ & $1.52 \pm 2.58(0-10)$ \\
Architectural distortion & $2.0 \pm 3.0(0-14)$ & $3.06 \pm 3.44(0-14)^{\dagger \ddagger}$ & $0.83 \pm 2.07(0-12)$ & $0.86 \pm 1.36(0-4)$ \\
GGO & $3.3 \pm 4.4(0-18)$ & $1.17 \pm 2.39(0-10)^{\dagger \ddagger}$ & $4.39 \pm 3.67(0-13)$ & $7.58 \pm 6.25(0-18)$ \\
Emphysema & $1.4 \pm 3.2(0-14)$ & $1.97 \pm 3.47(0-14)^{\dagger}$ & $0.57 \pm 1.95(0-11)$ & $1.55 \pm 3.90(0-14)$ \\
\hline
\end{tabular}

${ }^{\S} \mathrm{GGO}$, reticulation and honeycombing score max $=24$, traction bronchiectasis, architectural distortion and emphysema score max $=18$, score numbers are presented as mean $\pm \mathrm{SD}$ (min-max). P value $<0.05{ }^{\dagger}$ UIP vs. possible UIP, ${ }^{\ddagger}$ UIP vs. inconsistent with UIP. HRCT, highresolution computed tomography; UIP, usual interstitial pneumonia; GGO, ground glass opacity; N, number.

Cox regression analysis of the clinical features is presented in the Supplementary file (Table S1). The extent of traction bronchiectasis and the presence of emphysema independently associated with shortened survival in the multivariate Cox regression analysis (Table 5). The extent of emphysema did not associate with shortened survival in a multivariate analysis (data not shown). The possible UIP group had a longer median survival than the UIP patients in the unadjusted Kaplan-Meyer estimator analysis. The survival was also longer in patients without honeycombing, traction bronchiectasis, architectural distortion or emphysema (Figure 3 and Table S2).

\section{Correlation of the HRCT patterns with clinical features and PFT}

The extents of traction bronchiectasis, honeycombing, and architectural distortion had an inverse correlation with all PFT values at the time of diagnosis. The UIP pattern correlated with the extent of traction bronchiectasis, honeycombing, architectural distortion, and emphysema $(\mathrm{r}=0.401, \mathrm{P}<0.001 ; \mathrm{r}=0.742, \mathrm{P}<0.001 ; \mathrm{r}=0.491, \mathrm{P}<0.001$; $\mathrm{r}=0.233, \mathrm{P}<0.01$, respectively) showing an inverse correlation with GGO and reticulation $(\mathrm{r}=-0.522, \mathrm{P}<0.001$; $\mathrm{r}=-0.202, \mathrm{P}<0.05$, respectively). In addition, the extent of reticulation correlated with dyspnea $(r=0.195, \mathrm{P}<0.05)$ and 
Table 4 Univariate Cox regression analysis of the presence and extent of specific HRCT patterns in patients with idiopathic pulmonary fibrosis

\begin{tabular}{|c|c|c|c|c|c|c|}
\hline Radiological pattern & \multicolumn{3}{|c|}{ Presence } & \multicolumn{3}{|c|}{ Extent } \\
\hline Honeycombing & 2.017 & $1.297-3.137$ & 0.002 & 1.122 & $1.065-1.182$ & $<0.001$ \\
\hline Traction bronchiectasis & 1.757 & $1.078-2.863$ & 0.024 & 1.212 & $1.130-1.299$ & $<0.001$ \\
\hline Architectural distortion & 2.220 & $1.521-3.240$ & $<0.001$ & 1.160 & $1.094-1.231$ & $<0.001$ \\
\hline GGO & 0.800 & $0.549-1.164$ & 0.243 & 0.981 & $0.937-1.027$ & 0.412 \\
\hline Reticulation & 0.753 & $0.104-5.424$ & 0.778 & 1.023 & $0.954-1.098$ & 0.521 \\
\hline UIP-possible UIP, UIP & Reference & - & - & - & - & - \\
\hline Possible UIP & 0.644 & $0.428-0.970$ & 0.035 & - & - & - \\
\hline
\end{tabular}

$\mathrm{P}$ value $<0.05$ regarded as significant. HRCT, high-resolution computed tomography; Cl, confidence interval; HR, hazard ratio; GGO, ground glass opacity; UIP, usual interstitial pneumonia.

Table 5 Multivariate Cox regression analysis of the extent of the specific HRCT patterns and clinical features in patients with idiopathic pulmonary fibrosis

\begin{tabular}{lccc}
\hline Variable & HR & 95\% Cl & P \\
\hline Age & 1.058 & $1.030-1.087$ & - \\
Gender, female & Reference & - & 001 \\
Male & 1.946 & $1.087-3.483$ & 0.025 \\
DLCO\% & 0.956 & $0.938-0.973$ & $<0.001$ \\
DLCO absolute change in 12 months & 0.970 & $0.958-0.983$ & $<0.001$ \\
The presence of emphysema & 1.971 & $1.134-3.428$ & 0.016 \\
The extent of traction bronchiectasis & 1.227 & $1.088-1.385$ & 0.001 \\
The extent of architectural distortion & 0.984 & $0.871-1.112$ & 0.799 \\
\hline
\end{tabular}

$\mathrm{P}$ value $<0.05$ regarded as significant. HRCT, high-resolution computed tomography; HR, hazard ratio; Cl, confidence interval; DLCO\%, diffusion capacity to carbon monoxide percent predicted.

the extent of emphysema inversely correlated with cough $(\mathrm{r}=-0.306, \mathrm{P}<0.01)($ Table S3). Cough was more common in the possible UIP patients than in the UIP group. The number of hospitalizations due to respiratory reasons between the different radiological categories did not differ in the various groups (Table 1). However, more hospital visits due to respiratory reasons were observed among the non-UIP patients than in the UIP patients [49/64 (78\%) vs. $38 / 65$ (59\%), respectively, $\mathrm{P}=0.03]$. In addition, the hospital visits due to respiratory reasons correlated inversely with the extent of emphysema (Table S3). There were more eversmokers among men than women $(78 \%$ vs. $26 \%, \mathrm{P}<0.001)$ as well as among the patients with emphysema than those without emphysema ( $88 \%$ vs. $55 \%, \mathrm{P}=0.001)$. The men had more extensive honeycombing, architectural distortion, and emphysema than women, as did the ever-smokers in comparison to the non-smokers (Table S4).

\section{Discussion}

In this real-life study, we demonstrated that the extent of traction bronchiectasis correlated with shortened survival in IPF patients, even when adjusted for clinical features and another indicator of fibrosis. The presence of emphysema seemed to have a similar correlation as a categorical variable, whereas the emphysema score did not associate 
A

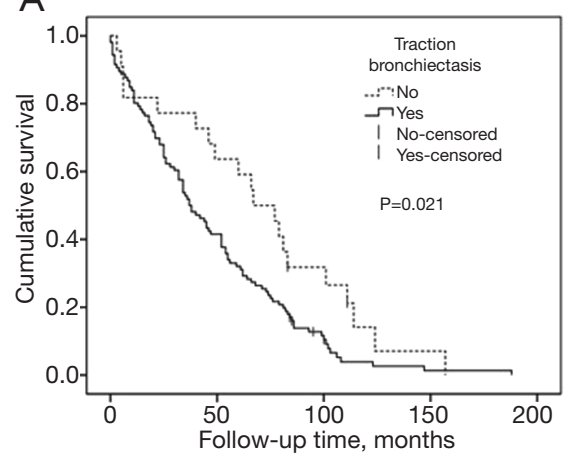

D

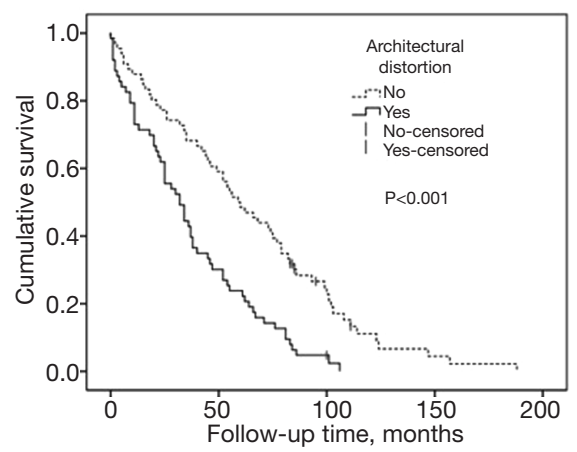

B

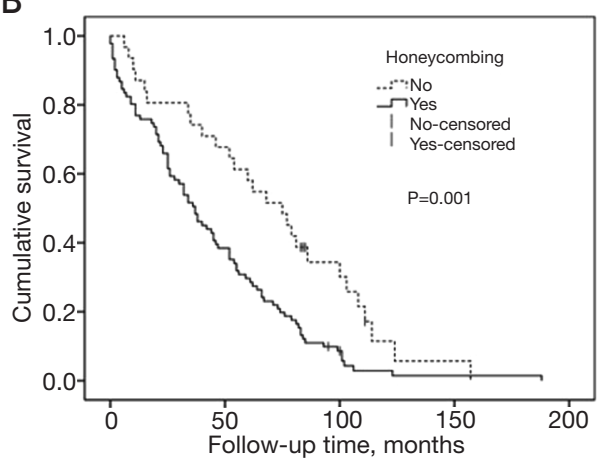

$\mathrm{E}$

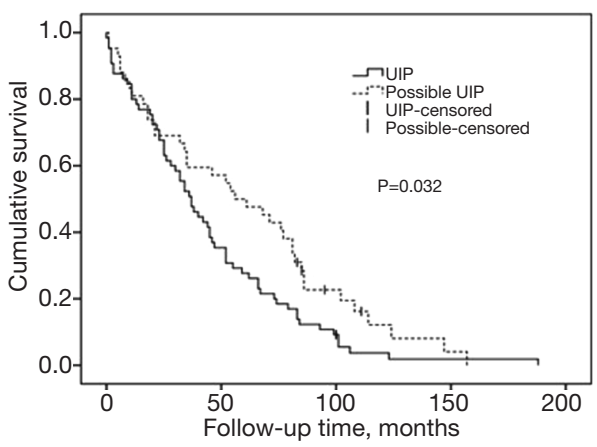

C

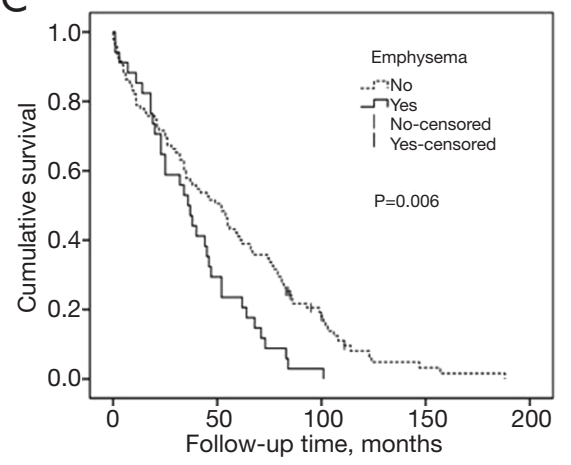

Figure 3 Unadjusted Kaplan-Meier survival curves in patients with idiopathic pulmonary fibrosis and the presence of specific HRCT patterns stratified by (A) traction bronchiectasis, (B) honeycombing, (C) emphysema, (D) architectural distortion, and (E) UIP $v s$. possible UIP pattern. Survival curves and P values represent the time to death or lung transplantation. HRCT, high-resolution computed tomography; UIP, usual interstitial pneumonia.

with survival. Furthermore, we found that the possible UIP patients had a longer survival in comparison to the UIP patients and that honeycombing and architectural distortion were associated with shortened survival. In addition, we made three novel findings. Firstly, the extent of several specific HRCT patterns, such as traction bronchiectasis, honeycombing, architectural distortion, and emphysema positively correlated with the UIP pattern. Secondly, the extent of reticulation correlated with dyspnea and the extent of emphysema inversely correlated with cough. Thirdly, there were a similar number of hospitalizations due to respiratory reasons in the different radiological categories. The radiological categorizations between the 2011 and 2018 international guidelines varied only marginally.

Although several previous studies have investigated specific HRCT-patterns, many of them have included fibrotic idiopathic interstitial pneumonia (IIP) patients rather than focusing on IPF patients only (15-17) or IPF patients were diagnosed according to an outdated international statement (18). Several studies focusing on IPF diagnosed according to the 2011 international statement, have found similar results regarding the correlation of survival with traction bronchiectasis, whereas the results of emphysema have been partly different (7-9,19-21). The two previous studies that have evaluated the correlation of specific HRCT patterns with survival in IPF analyzed fewer variables and moreover, the quantification of HRCT patterns was more modest than in our study $(7,8)$. In a study of 58 IPF patients, Sverzellati et al. discovered that the visually evaluated extent of traction bronchiectasis was associated with shortened survival in a univariate Cox regression analysis at baseline and in two years' follow up (9). Salisbury et al. found that the number of lung lobes with traction bronchiectasis was associated with an increased risk of death (8). Arcadu et al. did not detect any correlation between the presence of traction bronchiectasis and patient survival in a study of 350 IPF patients. However, in Arcadu's study, the extent of traction bronchiectasis was not analyzed, which could explain the contradictory result (7). Although Jacob et al. evaluated several 
HRCT patterns using automated CT analysis, traction bronchiectasis was evaluated visually. It was claimed that visually evaluated traction bronchiectasis was associated with shortened survival and a FVC decline during 12 months $(19,22)$. In more recent studies using quantitative CTanalysis, CT histogram values and vessel-related structures were associated with survival in IPF (23-25). Since traction bronchiectasis has not yet been studied with automated CT analysis methods, we believe that this present work is so far the most detailed study of traction bronchiectasis in IPF.

Several previous studies evaluating the correlation between pulmonary fibrosis, emphysema, and survival have included also patients with other interstitial lung diseases (ILDs) in addition to IPF, or they have not diagnosed IPF patients according to the current international IPF statement (26-28). In a study focusing on combined pulmonary fibrosis and emphysema (CPFE), there was no difference in survival between the patients with $\geq 10 \%$ emphysema and less fibrosis and those with more fibrosis and $<10 \%$ emphysema (20). Jacob et al. demonstrated that emphysema was associated with increased mortality in IPF patients, but independently the extent of emphysema did not predict mortality (21). Similarly, we did not find that the emphysema score would associate independently with survival even though the presence of emphysema seemed to correlate with shortened survival.

The pattern of architectural distortion described previously as "abnormal displacement of bronchi, vessels, fissures, or septa caused by diffuse or localized lung disease, particularly interstitial fibrosis" has not been studied previously in cases diagnosed according to the 2011 international IPF statement (29). We demonstrated that architectural distortion was associated with shortened survival and furthermore, it correlated with PFT values and several clinical features. Two previous studies with different study protocols have reported results that are in line with our findings. Arcadu et al. observed that the extent of "global assessment of interstitial abnormalities" was associated with increased mortality and correlated inversely with FVC and DLCO percent predicted (7). In a study of fibrotic idiopathic interstitial pneumonia (IIP) patients, the definite UIP patients with the overall extent of parenchymal abnormalities $>45 \%$ had shortened survival in comparison to those patients with an overall CT score $<45 \%$ (17).

Our result of the survival difference between the possible and definite UIP patterns is in line with previous studies $(7,8)$. Salisbury et al. showed that the possible UIP pattern was associated with longer survival in comparison to the definite UIP pattern and that the difference remained after adjustments (8). Arcadu et al. reported that the definite UIP pattern was associated with shortened survival in comparison to the possible and the inconsistent with UIP patterns (7). Controversially, a study of 42 IPF patients did not detect a survival difference between the definite UIP and the possible UIP patients, a finding which may be partly due to the small size of the cohort (6).

As in the previous studies, honeycombing was associated with shortened survival also in our study (6-8). Novelli et al. found that IPF patients with a honeycombing score $\geq 15 \%$ had worse survival than those who had a honeycombing score $<15 \%$ (6). In two more recent studies, honeycombing was associated with an increased risk of death in univariate Cox regression analyses $(7,8)$. In a quantitative CT-analysis study, the area of honeycombing was also associated with worse survival in IPF (30). Controversially, in a Japanese study, 43 IPF patients did not have honeycombing in HRCT at baseline and the emergence of honeycombing in the three years' follow-up did not affect patient survival. The small cohort size and relatively young mean age (64.5 years) of subjects examined in that study could partly explain the contradictory result (31).

The limitations of our study are those typically and often encountered in real-life studies. The study was retrospective, and the cohort size was relatively small. In addition, the CT protocol was variable, and the study was performed in one institution. The inter-observer agreement between the two radiologists for the UIP classifications in our study was moderate, which is similar to previous studies (32-34). However, the amount of suboptimal quality HRCT examinations $(31 \%)$ may have affected the radiologists' ability to interpret fine differences when evaluating the radiological classifications. In addition, there was a rather wide difference between the radiologists' experience in thoracic radiology (10 and 2 years of expertise, respectively). Nevertheless, in our study, the inter-observer agreement for specific HRCT patterns was good, which is also in line with previous studies $(6,9,15,16)$. Furthermore, not all of the material was available for the second HRCT analysis in 2018 .

\section{Conclusions}

In conclusion, several specific HRCT patterns were associated with shortened survival in IPF. The extent of traction bronchiectasis was one of the strongest patterns to be individually associated with shortened survival, which 
suggests that this pattern could be an important prognostic factor in IPF. These results may help in evaluating the risk of death in IPF patients.

\section{Acknowledgments}

The authors wish to thank Ewen MacDonald for providing assistance with the language.

Funding: The Foundation of the Finnish Anti-Tuberculosis Association to MM, RK; Väinö and Laina Kivi Foundation to MM; Center of Medicine and Clinical Research, Kuopio University Hospital to MM; the Research Foundation of Kuopio University Hospital to MM; a state subsidy of the Kuopio University Hospital to RK; the Research Foundation of Pulmonary Diseases to MM, RK; the Research Foundation of Northern Finland to RK, and the Jalmari and Rauha Ahokas Foundation to MM, RK.

\section{Footnote}

Reporting Checklist: The authors have completed the STROBE reporting checklist. Available at http://dx.doi. org/10.21037/jtd-20-1957

Data Sharing Statement: Available at http://dx.doi. org/10.21037/jtd-20-1957

Conflicts of Interest: All authors have completed the ICMJE uniform disclosure form (available at http://dx.doi. org/10.21037/jtd-20-1957). MM has received a consulting and lecture fees from Boehringer Ingelheim Finland Ltd. and congress travel costs from Roche and Boehringer Ingelheim Finland Ltd, outside the submitted work. HPK has received a lecture fee from Roche, outside the submitted work. SS owns stocks of Merck \& Co, Faron Paharmaceuticals, $3 \mathrm{M}$ Co, and CRISPR Therapeutics, outside the submitted work. MK has received an advisory board fee from GlaxoSmithKline, outside the submitted work. MP has received lecture and consulting fees from Boehringer-Ingelheim Finland Ltd. and a congress travel cost from Roche, outside the submitted work. RK has received a congress travel cost from Orion Pharma, lecture fees from Roche and Boehringer-Ingelheim Finland Ltd., and an advisory board fee from Boehringer Ingelheim Finland Ltd, outside the submitted work. TS declare no relationships with any companies, whose products or services may be related to the subject matter of the article.
Ethical Statement: The authors are accountable for all aspects of the work in ensuring that questions related to the accuracy or integrity of any part of the work are appropriately investigated and resolved. The study was conducted in accordance with the Declaration of Helsinki (as revised in 2013). Because of the retrospective nature of this study and the fact that most of the study subjects were already deceased, no informed consents for study inclusion were collected. The study protocol was approved by the Research Ethics Committee of the Northern Savo Hospital District (statement 17/2013) and by the National Institute for Health and Welfare (Dnro THL/1052/5.05.01/2013).

Open Access Statement: This is an Open Access article distributed in accordance with the Creative Commons Attribution-NonCommercial-NoDerivs 4.0 International License (CC BY-NC-ND 4.0), which permits the noncommercial replication and distribution of the article with the strict proviso that no changes or edits are made and the original work is properly cited (including links to both the formal publication through the relevant DOI and the license). See: https://creativecommons.org/licenses/by-nc-nd/4.0/.

\section{References}

1. Raghu G, Collard HR, Egan JJ, et al. An official ATS/ ERS/JRS/ALAT statement: Idiopathic pulmonary fibrosis: Evidence-based guidelines for diagnosis and management. Am J Respir Crit Care Med 2011;183:788-824.

2. Raghu G, Lynch D, Godwin JD, et al. Diagnosis of idiopathic pulmonary fibrosis with high-resolution CT in patients with little or no radiological evidence of honeycombing: Secondary analysis of a randomised, controlled trial. Lancet Respir Med 2014;2:277-84.

3. Salisbury ML, Xia M, Murray S, et al. Predictors of idiopathic pulmonary fibrosis in absence of radiologic honeycombing: A cross sectional analysis in ILD patients undergoing lung tissue sampling. Respir Med 2016;118:88-95.

4. Gruden JF, Panse PM, Gotway MB, et al. Diagnosis of usual interstitial pneumonitis in the absence of honeycombing: Evaluation of specific CT criteria with clinical follow-up in 38 patients. AJR. Am J Roentgenol 2016;206:472.

5. Raghu G, Remy-Jardin M, Myers JL, et al. Diagnosis of Idiopathic Pulmonary Fibrosis. An Official ATS/ERS/JRS/ ALAT Clinical Practice Guideline. Am J Respir Crit Care 
Med 2018;198:e44-e68.

6. Novelli F, Tavanti L, Cini S, et al. Determinants of the prognosis of idiopathic pulmonary fibrosis. Eur Rev Med Pharmacol Sci 2014;18:880.

7. Arcadu A, Byrne SC, Pirina P, et al. Correlation of pulmonary function and usual interstitial pneumonia computed tomography patterns in idiopathic pulmonary fibrosis. Respir Med 2017;129:152-7.

8. Salisbury ML, Tolle LB, Xia M, et al. Possible UIP pattern on high-resolution computed tomography is associated with better survival than definite UIP in IPF patients. Respir Med 2017;131:229-35.

9. Sverzellati N, Silva M, Seletti V, et al. Stratification of long-term outcome in stable idiopathic pulmonary fibrosis by combining longitudinal computed tomography and forced vital capacity. Eur Radiol 2020;30:2669-79.

10. Kärkkäinen M, Kettunen H, Nurmi H, et al. Effect of smoking and comorbidities on survival in idiopathic pulmonary fibrosis. Respir Res 2017;18:160.

11. Kärkkäinen $M$, Nurmi H, Kettunen H, et al. Underlying and immediate causes of death in patients with idiopathic pulmonary fibrosis. BMC Pulm Med 2018;18:69.

12. Kärkkäinen $M$, Kettunen H, Nurmi H, et al. Comparison of disease progression subgroups in idiopathic pulmonary fibrosis. BMC Pulm Med 2019;19:228.

13. Nurmi HM, Kettunen H, Suoranta S, et al. Several highresolution computed tomography findings associate with survival and clinical features in rheumatoid arthritis-associated interstitial lung disease. Respir Med 2018;134:24-30.

14. Ley B, Collard HR, King J, et al. Clinical course and prediction of survival in idiopathic pulmonary fibrosis. Am J Respir Crit Care Med 2011;183:431-40.

15. Edey AJ, Devaraj AA, Barker RP, et al. Fibrotic idiopathic interstitial pneumonias: HRCT findings that predict mortality. Eur Radiol 2011;21:1586-93.

16. Lee HY, Lee KS, Jeong YJ, et al. High-Resolution CT findings in fibrotic idiopathic interstitial pneumonias with little honeycombing: Serial changes and prognostic implications. AJR Am J Roentgenol 2012;199:982-9.

17. Romei C, Tavanti L, Sbragia P, et al. Idiopathic interstitial pneumonias: Do HRCT criteria established by ATS/ ERS/JRS/ALAT in 2011 predict disease progression and prognosis? Radiol Med 2015;120:930-40.

18. Lynch DA, Godwin JD, Safrin S, et al. High-resolution computed tomography in idiopathic pulmonary fibrosis: diagnosis and prognosis. Am J Respir Crit Care Med 2005;172:488.
19. Jacob J, Bartholmai BJ, Rajagopalan S, et al. Mortality prediction in idiopathic pulmonary fibrosis: Evaluation of computer-based CT analysis with conventional severity measures. Eur Respir J 2017;49:1601011.

20. Ryerson CJ, Hartman T, Elicker BM, et al. Clinical features and outcomes in combined pulmonary fibrosis and emphysema in idiopathic pulmonary fibrosis. Chest 2013;144:234-40.

21. Jacob J, Bartholmai BJ, Rajagopalan S, et al. Functional and prognostic effects when emphysema complicates idiopathic pulmonary fibrosis. Eur Respir J 2017;50:1700379.

22. Jacob J, Bartholmai BJ, Rajagopalan S, et al. Predicting outcomes in idiopathic pulmonary fibrosis using automated CT analysis. Am J Respir Crit Care Med 2018;198:767-76.

23. Torrisi SE, Palmucci S, Stefano A, et al. Assessment of survival in patients with idiopathic pulmonary fibrosis using quantitative HRCT indexes. Multidiscip Respir Med 2018;13:43.

24. Loeh B, Brylski LT, von der Beck D, et al. Lung CT densitometry in idiopathic pulmonary fibrosis for the prediction of natural course, severity, and mortality. Chest 2019;155:972-81.

25. Jacob J, Bartholmai BJ, van Moorsel CHM, et al. Longitudinal prediction of outcome in idiopathic pulmonary fibrosis using automated CT analysis. Eur Respir J 2019;54:1802341.

26. Kurashima K, Takayanagi N, Tsuchiya N, et al. The effect of emphysema on lung function and survival in patients with idiopathic pulmonary fibrosis. Respirology 2010;15:843-8.

27. Jankowich MD, Rounds S. Combined pulmonary fibrosis and emphysema alters physiology but has similar mortality to pulmonary fibrosis without emphysema. Lung 2010;188:365-73.

28. Todd NW, Jeudy J, Lavania S, et al. Centrilobular emphysema combined with pulmonary fibrosis results in improved survival. Fibrogenesis Tissue Repair 2011;4:6.

29. Hansell DM, Bankier AA, MacMahon H, et al. Fleischner Society: glossary of terms for thoracic imaging. Radiology 2008;246:697-722.

30. Nakagawa H, Ogawa E, Fukunaga K, et al. Quantitative CT analysis of honeycombing area predicts mortality in idiopathic pulmonary fibrosis with definite usual interstitial pneumonia pattern: A retrospective cohort study. PLoS One 2019;14:e0214278.

31. Yamauchi H, Bando M, Baba T, et al. Clinical course and changes in high-resolution computed tomography findings 
in patients with idiopathic pulmonary fibrosis without honeycombing. PloS one 2016;11:e0166168.

32. Aziz ZA, Wells AU, Hansell DM, et al. HRCT diagnosis of diffuse parenchymal lung disease: inter-observer variation. Thorax 2004;59:506-11.

33. Sumikawa H, Johkoh T, Colby TV, et al. Computed

Cite this article as: Mononen ME, Kettunen HP, Suoranta SK, Kärkkäinen MS, Selander TA, Purokivi MK, Kaarteenaho RL. Several specific high-resolution computed tomography patterns correlate with survival in patients with idiopathic pulmonary fibrosis. J Thorac Dis 2021;13(4):2319-2330. doi: 10.21037/jtd-201957 tomography findings in pathological usual interstitial pneumonia: Relationship to survival. Am J Respir Crit Care Med 2008;177:433-9.

34. Widell J, Lidén M. Interobserver variability in highresolution CT of the lungs. Eur J Radiol Open 2020;7:100228. 


\section{Supplementary}

Table S1 Univariate Cox regression analysis of the clinical features in patients with idiopathic pulmonary fibrosis

\begin{tabular}{lccc}
\hline & HR & 95\% Cl & P \\
\hline Age & 1.039 & $1.018-1.061$ & $<.001$ \\
FVC\% & 0.981 & $0.970-0.992$ & $<.001$ \\
FEV1\% & 0.977 & $0.966-0.989$ & $<.001$ \\
DLCO\% & 0.968 & $0.956-0.980$ & 0.001 \\
FVC absolute change in 12 months & 0.983 & $0.971-0.995$ & 0.006 \\
DLCO absolute change in 12 months & 0.988 & $0.977-0.998$ & 0.020 \\
Gender, female & Reference & & 0.022 \\
Male & 1.619 & $1.071-2.447$ & 0.433 \\
Ever-smoker & 1.162 & $0.798-1.692$ & 0.693 \\
Dyspnea & 1.088 & $0.716-1.653$ & 0.023 \\
Cough & 0.654 & $0.454-0.943$ & 0.368 \\
Oxygen therapy & 0.843 & $0.580-1.224$ & \\
\hline
\end{tabular}

$\mathrm{P}$ value $<0.05$ regarded as significant. HRCT, high-resolution computed tomography; HR, hazard ratio; $\mathrm{Cl}$, confidence interval; FVC\%, forced vital capacity percent predicted; FEV1\%, forced expiratory volume in one second percent predicted; DLCO\%, diffusion capacity to carbon monoxide percent predicted.

Table S2 Median survival in patients with IPF in the presence of specific HRCT patterns according to the unadjusted Kaplan-Meier estimator analysis

\begin{tabular}{lcc}
\hline Radiological pattern & Median survival in months $(95 \% \mathrm{Cl})$ & Log Rank P \\
\hline Honeycombing: no/yes & $75(54.3-95.7) / 37(27.7-46.3)$ & 0.001 \\
Traction bronchiectasis: no/yes & $67(45.2-88.8) / 37(28.3-45.7)$ & 0.021 \\
Emphysema: no/yes & $52(37.2-66.8) / 36(27.4-44.6)$ & 0.006 \\
Architectural distortion: no/yes & $59(43.1-74.9) / 32(22.0-42.0)$ & $<0.001$ \\
Possible UIP/UIP & $56(29.5-82.5) / 37(28.0-46.0)$ & 0.032 \\
\hline
\end{tabular}

$\mathrm{P}$ value $<0.05$ regarded as significant. IPF, idiopathic pulmonary fibrosis; HRCT, high resolution computed tomography; Cl, confidence interval; UIP, usual interstitial pneumonia. 
Table S3 Correlations between the extent of HRCT patterns and selected features in patients with IPF according to Spearman's correlation coefficient

\begin{tabular}{|c|c|c|c|c|c|c|}
\hline & $\begin{array}{c}\text { Traction } \\
\text { bronchiectasis Score }\end{array}$ & $\begin{array}{l}\text { Honeycombing } \\
\text { Score }\end{array}$ & $\begin{array}{c}\text { Architectural } \\
\text { distortion Score }\end{array}$ & $\begin{array}{l}\text { Reticulation } \\
\text { Score }\end{array}$ & $\begin{array}{l}\text { GGO } \\
\text { Score }\end{array}$ & $\begin{array}{c}\text { Emphysema } \\
\text { Score }\end{array}$ \\
\hline FVC\% & $-0.187^{\star}$ & $-0.225^{\star}$ & $-0.193^{\star}$ & $-0.281^{\star \star}$ & -0.059 & $0.240^{\star *}$ \\
\hline DLCO\% & $-0.347^{\star \star \star}$ & $-0.284^{\star *}$ & $-0.298^{\star \star}$ & $-0.279^{\star \star}$ & -0.039 & -0.083 \\
\hline DLCO12 & 0.197 & $0.232^{*}$ & 0.177 & -0.081 & -0.046 & 0.023 \\
\hline Ever-smoker & -0.064 & $0.206^{*}$ & $0.207^{*}$ & -0.141 & -0.106 & $0.280^{* *}$ \\
\hline Gender & 0.127 & $0.289^{\star \star}$ & $0.253^{\star \star}$ & -0.051 & -0.081 & $0.208^{*}$ \\
\hline $\mathrm{HC}$ & $0.645^{\star \star \star}$ & 1.000 & $0.680^{\star \star \star}$ & -0.105 & $-0.364^{\star \star \star}$ & $0.205^{\star}$ \\
\hline Ret & $0.180^{\star}$ & -0.105 & -0.147 & 1.000 & $0.443^{\star \star \star}$ & $-0.284^{\star \star}$ \\
\hline Em & 0.003 & $0.205^{\star}$ & $0.289^{\star \star}$ & $-0.284^{\star \star}$ & $-0.224^{*}$ & 1.000 \\
\hline TX & 1.000 & $0.645^{\star \star \star}$ & $0.617^{\star \star \star}$ & $0.180^{*}$ & $-0.184^{*}$ & 0.003 \\
\hline AD & $0.617^{\star \star \star}$ & $0.680^{\star \star \star}$ & 1.000 & -0.147 & $-0.249^{\star \star}$ & $0.289^{\star \star}$ \\
\hline
\end{tabular}

${ }^{*}, \mathrm{P}<0.05 ;{ }^{* \star}, \mathrm{P}<0.01 ;{ }^{* \star}, \mathrm{P}<0.001 ;{ }^{\dagger}$, classified change in six months; ${ }^{\ddagger}$, absolute change in 12 months; ${ }^{\S}$, classified change in 12 months. IPF, idiopathic pulmonary fibrosis; HRCT, high resolution computed tomography; GGO, ground glass opacity; FVC\%, forced vital capacity percent predicted; FEV1\%, forced expiratory volume in one second percent predicted; DLCO\%, diffusion capacity to carbon monoxide percent predicted; ReHosp, hospitalization due to respiratory reasons (yes/no); UIP, usual interstitial pneumonia; HC, honeycombing; Ret, reticulation; Em, emphysema; TX, traction bronchiectasis; AD, architectural distortion.

Table S4 The extent of specific HRCT patterns in patients with IPF categorized according to gender and different smoking habits

\begin{tabular}{lcccc}
\hline Feature & Female & Male & Non-smoker & Ever-smoker \\
\hline Reticulation & $9.06 \pm 2.6(3-13)$ & $8.89 \pm 3.3(0-19)$ & $9.40 \pm 2.3(4-13)$ & $8.73 \pm 3.4(0-19)$ \\
Traction bronchiectasis & $2.83 \pm 2.6(0-10)$ & $3.68 \pm 2.9(0-13)$ & $3.74 \pm 3.1(0-13)$ & $3.27 \pm 2.8(0-12)$ \\
Honeycombing & $2.12 \pm 2.8(0-10)$ & $4.18 \pm 3.6(0-14)^{\star}$ & $2.63 \pm 3.2(0-14)$ & $4.03 \pm 3.6(0-13)^{\star}$ \\
Architectural distortion & $0.89 \pm 1.7(0-6)$ & $2.34 \pm 3.2(0-14)^{\star}$ & $1.20 \pm 2.1(0-8)$ & $2.34 \pm 3.3(0-14)^{\star}$ \\
GGO & $4.13 \pm 5.4(0-18)$ & $2.94 \pm 3.9(0-16)$ & $4.05 \pm 4.9(0-18)$ & $2.91 \pm 4.0(0-16)$ \\
Emphysema & $0.37 \pm 1.1(0-4)$ & $1.82 \pm 3.6(0-14)^{\star}$ & $0.50 \pm 2.0(0-12)$ & $1.98 \pm 3.6(0-14)^{\star}$ \\
\hline
\end{tabular}

Score numbers are presented as mean $\pm \mathrm{SD}$ (min-max). ${ }^{*}, \mathrm{P}<0.05$. IPF, idiopathic pulmonary fibrosis; HRCT, high resolution computed tomography; GGO, ground glass opacity. 